\title{
Notas en pos de la regulación jurídica del balance social cooperativo en Cuba
}

\author{
Reflections on legal regulation \\ of Cooperative Social Balance in Cuba
}

Lien Soto Alemán ${ }^{1}$

Universidad de la Habana (Cuba)

Sumario: I. Introducción. II. Comportamiento de los principios del cooperativismo moderno en cooperativas de diferentes sectores. III. Experiencias de Balance Social Cooperativo en Cuba. IV. A favor de un marco jurídico para la utilización del balance social cooperativo. V. Conclusiones. VI. Bibliografía.

Summary: I. Introduction. II. Application of the principles of the modern cooperativism in different sectors of cooperatives. III. Experiences on cooperative social balance in Cuba. IV. Supporting a legal framework to apply Cooperative Social Balance V. Conclusions. Vl. Bibliography.

Resumen: En el ámbito cooperativo la responsabilidad social se percibe como una alternativa que enaltece la ética y la filosofía cooperativa, en la práctica y cumplimiento de los principios y valores de estas. Se ha elevado a rango de principio en algunas legislaciones como la cubana. En el presente trabajo se muestran los resultados de la evaluación del cumplimiento de los principios del cooperativismo en algunas cooperativas seleccionadas, así como se analizan algunas experiencias de Balance Social Cooperativo desarrollados en cooperativas del país. Se concluye con reflexiones en torno a los presupuestos teóricos y jurídicos que debe informar una futura regulación del Balance Social Cooperativo en Cuba.

Palabras clave: Cooperativa, identidad, responsabilidad social, balance social.

Abstract: In the cooperative environment, the social responsibility is perceived like an alternative that ennobles the ethics and the cooperative philosophy, in the practice and fulfillment of its principles and values. In some

1 Máster en Derecho de la Economía. Profesora Auxiliar de Derecho Económico. Facultad de Derecho. Universidad de La Habana, e-mail: lien@lex.uh.cu. 
legislations like in the cuban legislation has risen to the range of a principle. Currently work analyzes he results of the evaluation of the executions of the principles of the cooperativism are shown in some selected cooperatives, as well as some experiences regarding Cooperative Social Balance, developed in cooperatives of the country. Finally, the theoretical-juridical premises that should be informed by a future regulation of the Cooperative Social Balance are analyzed.

Keywords: Cooperative, identity, social responsibility, social balance. 


\section{Introducción}

El movimiento cooperativo cubano tradicional se integra por las cooperativas agropecuarias, en sus dos formas, las de producción y las de créditos y servicios², consignadas desde la Ley de Reforma Agraria del 17 de mayo de 1959, las que en conjunto han constituido una forma de producción socialista y propiciado además, el desarrollo social de la vida campesina como uno de los principios de la política agraria en el país. El marco regulatorio está contenido en la Ley No. 95 de Cooperativas de Producción Agropecuaria y de Créditos y Servicios que derogó la Ley $36 / 82^{3}$. Comparten espacio, aunque con una realidad jurídica diferente las Unidades Básicas de Producción Cooperativa ${ }^{4}$, las que constituyen un patrimonio con personalidad jurídica, en este caso compuesto por los bienes agropecuarios y los frutos de la tierra recibida en usufructo, tierra que se mantiene bajo la propiedad del Estado 5 .

Con la «actualización del modelo económico» las cooperativas del país se han extendido a sectores distintos del tradicionalmente reconocido y en el año 2012 vio la luz el Decreto Ley 305 «De las cooperativas no agropecuarias» ${ }^{6}$ y su Reglamento mediante el Decreto 309/12 ${ }^{7}$.

Al cierre del año 2016 se habían constituido en el país 383 CNoA, superior en poco más de una docena a 2015 (367). Este ritmo de crecimiento constituye el menor desde que en el país se impulsó este sector ${ }^{8}$, aunque al menos, es coherente con la estrategia anunciada por el gobierno de avanzar de manera gradual en la consolidación de esa forma de gestión, para rectificar errores antes de generalizar el experimento.

2 En lo adelante CPA y CCS, respectivamente.

3 Ley No. 95 De las Cooperativas de Producción Agropecuaria y de Créditos y Servicios, de fecha 2 de noviembre de 2002. Gaceta Oficial No. 72 Ordinaria de 29 de noviembre de 2002.

4 Decreto Ley No. 142 Sobre las Unidades Básicas de Producción Cooperativa, de fecha 20 de septiembre de 1993. Gaceta Oficial de la República de Cuba No. 6 Extraordinaria de 21 de septiembre de 1993. En lo adelante UBPC.

5 Más ampliamente en relación a las cooperativas del sector agropecuario véase: Armando Nova González, Las cooperativas agropecuarias en Cuba: 1959-presente, en Cooperativas y Socialismo: Una mirada desde Cuba, comp. por Camila Piñeiro Harnecker, (La Habana: Ed. Caminos, 2011), 321-336.

6 Decreto Ley No. 305 De las Cooperativas no Agropecuarias, de fecha 15 de noviembre de 2012. Gaceta Oficial No.53 Extraordinaria de 11 de diciembre de 2012.

7 Decreto No. 309 Reglamento de las Cooperativas no Agropecuarias de primer grado, de fecha 28 de noviembre de 2012. Gaceta Oficial de la República de Cuba No. 53 Extraordinaria de 11 de diciembre de 2012.

8 En 2013 se constituyeron 198, y al año siguiente 147. Sitio oficial de la ONEI, disponible en: http://www.one.cu/, acceso el 20 de febrero de 2016. 
En el contexto descrito, se asume el papel complementario del sector cooperativo respecto de la empresa estatal9; y se entiende que las acciones a desarrollar por las cooperativas están dirigidas a potenciar el desarrollo local del entorno donde insertan su gestión. Entendido el territorio no como espacio abstracto e indiferenciado sino como actor fundamental de desarrollo, integrado no sólo por el medio físico sino por los actores sociales y sus organizaciones, las instituciones locales, los cuales además del capital económico y financiero incorporan las diferentes dimensiones del capital humano, el capital social e institucional y el capital natural'10. Es por ello que el tejido económico en la base tiene en las cooperativas cubanas actores fundamentales y de ellas se espera una mayor responsabilidad social.

Sin embargo, cuando se analizan los Lineamientos actualizados de la Política Económica y Social aprobados en abril de 2016 en el VII Congreso del Partido, aunque se señala expresamente, en los numerales 52 y 99, el término responsabilidad social en todas las formas de gestión ${ }^{11}$, ello tiene lugar de forma restrictiva y enfocado exclusivamente al cumplimiento de obligaciones tributarias, constituyendo esto una forma indirecta de responsabilidad social ${ }^{12}$.

9 Lineamiento 2- «El modelo de gestión reconoce y promueve, además de la empresa estatal socialista, que es la forma principal en la economía nacional, las modalidades de inversión extranjera previstas en la ley (empresas mixtas, contratos de asociación económica internacional, entre otras), las cooperativas, los agricultores pequeños, los usufructuarios, los arrendatarios, los trabajadores por cuenta propia y otras formas, todas las que, en conjunto, deben contribuir a elevar la eficiencia». Lineamientos de la Política Económica y Social del Partido y la Revolución», p 10. Disponible en: http://www. cubadebate.cu/noticias/2011/05/09/descargue-en-cubadebate-los-lineamientos-de-lapolitica-economica-y-social-pdf/. Acceso el 12 de enero 2017.

10 Vid. Francisco Albuquerque Llorens, «Desarrollo económico local y descentralización en América Latina», Revista de la CEPAL, No. 82 (2004): 22.

11 Lineamiento 52: Fomentar la cultura tributaria y la responsabilidad social de la población, entidades y formas de gestión no estatales del país, en el cumplimiento cabal de las obligaciones tributarias, para desarrollar el valor cívico de contribución al sostenimiento de los gastos sociales y altos niveles de disciplina fiscal.

Lineamiento 99. Continuar desarrollando el marco jurídico y regulatorio que propicie la introducción sistemática y acelerada de los resultados de la ciencia, la innovación y la tecnología en los procesos productivos y de servicios, y el cumplimiento de las normas de responsabilidad social y medioambiental establecidas. Disponible en: http://www.cubadebate.cu/noticias/2016/05/09/descargue-en-cubadebate-los-lineamientos-de-la-politica-economica-y-social-pdf/. Acceso el 12 de enero 2017.

12 José Luis Alfonso Alemán et al., "La Gestión y el Balance Social en la Empresa Cooperativa Cubana. Caso de Estudio: CPA 14 de junio», Revista Contabilidad y Auditoría, No. 29 (2009): 71. 
En tal sentido en el contexto actual resulta relevante contar con herramientas de medición de la incidencia social de estas figuras, a fin de evaluar su impacto social y el grado de cumplimiento de los principios cooperativos. Lo que armonizaría con los llamados contenidos en el Plan para una década cooperativa, Visión $2020^{13}$ de la Alianza Cooperativa Internacional que plantea la necesidad de innovar en la contabilidad no financiera del sector cooperativo.

En efecto, contabilizar el desempeño no financiero de las entidades ha sido una reclamación exponencial de la sociedad en las últimas décadas por lo que variadas han sido las formas y modelos adoptados, desde las memorias de sostenibilidad, los indicadores del Global Reporting Iniciative (GRI), la contabilidad de triple raya final (TBI), el método de «tarjeta de puntuación del balance», por solo citar algunos. En el ámbito cooperativo se ha trabajado arduamente en la concepción e instrumentación del balance social cooperativo ${ }^{14}$.

El investigador SAN BARTOLOME, en el marco del Foro sobre Balance Social Cooperativo y Gobernabilidad de la ACI AMERICAS, celebrado en 2010 en la ciudad de Buenos Aires, planteó que el BSCoop es una herramienta de la gestión socioeconómica que les facilita a las cooperativas medirse y rendir cuentas a los asociados especialmente y a todos los demás grupos de interés que están impactados por su accionar en relación con el cumplimiento de su propia esencia o identidad, es decir desde los valores y los principios cooperativos ${ }^{15}$.

Este concepto de BSCoop apunta dos elementos claves, de una parte el «fin» que persigue y de otro, el «contenido» de lo que expresa. Hay que tener en cuenta que hablar de cooperativas es hablar de organizaciones de intereses colectivos, donde como afirma MUGARRA las personas se unen para enfrentar sus problemas y buscar soluciones conjuntas, son entidades que buscan cumplir con un fin social siendo el aspecto económico un medio de gestión y no la finalidad última de su labor. Por ello el fin del balance social es la medición de la gestión social de la cooperativa y la rendición de cuentas a los grupos

13 ALIANZA COOPERATIVA INTERNACIONA, Plan para una década cooperativa, Visión 2020, 2013. Plan para una década cooperativa, Visión 2020, 2013, [on line], disponible en: http://www.aciamericas.coop/Plan-para-una-Decada-Cooperativa. Acceso el 20 de octubre de 2016.

14 En lo adelante BSCoop.

15 Juan Carlos San Bartolomé, Balance social cooperativo: una responsabilidad intrínseca de la empresa cooperativa. Foro Balance Social Cooperativo y Gobernabilidad. XVII Conferencia Regional de ACI AMERICAS. (Conferencia, Buenos Aires, Argentina, 22 de noviembre de 2010). 
de interés de esta, el contenido a su vez indica el nivel de cumplimiento de los principios y valores de la cooperativa ${ }^{16}$.

En relación a la regulación versus voluntariedad del BSCoop a nivel internacional, existen dos tendencias que van delineándose en la misma medida que se desarrollan las formas de economía social en cada uno de los países. De una parte Europa y los países nórdicos, donde cada vez más se reclama por las organizaciones no gubernamentales y la sociedad civil la regulación de instrumentos de evaluación del desempeño social de estas entidades con carácter obligatorio, incorporándose de hecho al marco regulatorio de estos países, y de otro América, donde en su generalidad no resulta obligatorio para las empresas cumplir con los indicadores sociales ni reportar su cumplimiento, salvo algunas excepciones como Ecuador y Paraguay. No obstante, en el sector cooperativo cada vez más se incrementa la presión de los diferentes actores sociales y del propio movimiento cooperativo regional para que sea utilizado el balance social como instrumento obligatorio por este sector. De hecho la Ley Marco para las cooperativas de América Latina ${ }^{17}$, que funge como marco referencial para las legislaciones nacionales de la región, dispone en el artículo 48 sobre Memoria y estados contables: «A la fecha de cierre del ejercicio el consejo de administración redactará una memoria sobre la gestión realizada la cual, juntamente con los estados contables y un balance que demuestre el desempeño de la cooperativa en el campo social, será sometida a la asamblea con informes de la junta de vigilancia y del auditor»; evidenciando con ello la importancia de rendir información social de conformidad con la naturaleza económica y social de la cooperativa.

En Ecuador, la imposición del balance social con carácter obligatorio como herramienta de evaluación del grado de cumplimiento de los principios y los objetivos sociales que informan las cooperativas, mutuales y demás empresas solidarias, tiene lugar mediante la Ley de Eco-

16 Más ampliamente sobre el tema véase Aitziber Mugarra Elorriaga, «Proyecto de Balance de Identidad Cooperativa», en Anuario de Estudios Cooperativos, No. 199 (Universidad de Deusto, Bilbao, 1999): 303-309; y de la propia autora: «Responsabilidad y balance social hoy en día: un reto para las cooperativas», Ciriec. Revista Jurídica de Economía Social y Cooperativa, No. 39 (2001): 27-50; y «Modelos de Balance Social: Criterios clasificatorios», Instituto de Estudios Cooperativos COLECCION DE CASOS, (Universidad de Deusto, Bilbao, 2009):128; además, Adil Belhouari et al., «La responsabilidad social de las empresas: ¿Un nuevo valor para las cooperativas?». Ciriec. Revista Jurídica de Economía Social y Cooperativa, No. 53 (2005): 191-211.

17 Ley Marco para las cooperativas de América Latina/ Alianza Cooperativa Internacional para las Américas. - 1a ed.- San José, Costa Rica: Alianza Cooperativa Internacional para las Américas, 2009. 
nomía Popular y Solidaria del sistema financiero, de 201118; al respecto recalca en el fin del balance social para la preservación de la identidad cooperativa. Para aquellas entidades que no entreguen el reporte de balance social se prevén sanciones de carácter administrativo como la multa pecuniaria y la suspensión temporal o definitiva del Registro General de Cooperativas. Este alcance punitivo de la normativa ecuatoriana es novedosa en el ámbito latinoamericano.

En Paraguay existe la Resolución N. ${ }^{\circ} 4109 / 2009^{19}$, sobre el BSCoop, emitida por el Instituto Nacional de Cooperativismo (INCOOP) que obliga a las cooperativas (según clasificación que establece la propia normativa «A», «B», «C $)$ a la presentación del balance social; sin embargo, según un informe consultado de la Oficina Subregional de la OIT para el cono sur de América Latina continúan existiendo dificultades en la recolección de datos para analizar el impacto de las cooperativas en el sector social, debido en parte a que la información remitida no se encuentra homogéneamente cuantificada 20 . A juicio de esta autora, ello obedece a la ausencia de indicadores sociales en el país, estandarizados y aplicables a las cooperativas.

En definitivas, la tendencia en Latinoamérica sobre regulación del balance social ha sido, en general, de aplicación voluntaria con incentivos para fomentar su aplicación, aunque cada vez más se aboga por la inclusión y exigencia obligatoria de información social; llegando a establecerse con carácter obligatorio en Paraguay y Ecuador, aunque no se estandarizan indicadores sociales que permitan brindar información homogénea. Un escenario distinto tiene lugar en el ámbito Europeo donde existen normativas que además de exigir con carácter obligato-

18 Cfr. DISPOSICION GENERAL SEGUNDA: - Las organizaciones sujetas a esta Ley incorporarán en sus informes de gestión, el balance social que acreditará el nivel de cumplimiento de los principios y sus objetivos sociales, en cuanto a la preservación de su identidad, su incidencia en el desarrollo social y comunitario, impacto ambiental, educativo y cultural. Vid. - Ley Orgánica de la Economía Popular y Solidaria del Sistema Financiero Popular y Solidario» de Ecuador, de 28 de abril de 2011. Registro Oficial 444 de 10 de mayo de 2011. Disponible en: http://www.oficial.ec/decreto-679-reformesereglamento-general-ley-organica-economia-popular-solidaria. Acceso el 25 de diciembre de 2016.

19 La Resolución aprobó más que indicadores sociales un modelo de uso obligatorio por las cooperativas. Se encuentra disponible en el sitio: www.incoop.gov.py. Acceso el 22 de enero de 2016.

20 Leticia Carosini, Visión panorámica del sector cooperativo en Paraguay. Una importante contribución al desarrollo nacional, La Paz, OIT, Oficina de la OIT para los Países Andinos, p. 65 [on line], 2012, disponible en:

http://www.ilo.org/santiago/publicaciones/WCMS_191115/lang--es/index.htm. Acceso el 10 de agosto de 2016. 
rio la información social (Francia, Italia, y Reino Unido), se establecen indicadores concretos (Reino Unido), así como existen órganos encargados de la supervisión y control de las cooperativas que deben dar seguimiento al impacto de estas entidades en sus comunidades, con facultad sancionadora (Reino Unido) ${ }^{21}$.

El caso español cuenta además con la herramienta RSE.COOP elaborada por CEPES22, con un elevado grado de seguimiento entre las cooperativas que están apostando por este tema, y que como afirman CASTILLA y GALLARDO se inserta en un denominador más genérico: revelación social o comunicación social al incluir todo tipo de comunicaciones efectuadas con el objetivo de dar a conocer públicamente aspectos de la responsabilidad social cooperativa ${ }^{23}$.

\section{Comportamiento de los principios del cooperativismo en cooperativas de diferentes sectores en Cuba}

Desde el Derecho, los principios han sido generalmente aceptados como normas de comportamiento ético y reglas de conducta. La Ley 95/02 de Cooperativas de Producción Agropecuaria y de Créditos y Servicios ${ }^{24}$, establece en su artículo 3 los principios generales sobre los cuales las cooperativas del sector agrario deben regirse. En tal sentido reconoce que la incorporación y permanencia de los miembros de las cooperativas es absolutamente voluntaria (la voluntariedad); que todos los miembros trabajan y aúnan sus esfuerzos para el uso racional de los suelos y bienes agropecuarios, propiedad o en usufructo de las cooperativas o de los cooperativistas (cooperación y ayuda mutua); que todos sus miembros conocen, cumplen y acatan conscientemente, las disposiciones de la Ley, sus reglamentos, los acuerdos de la Asamblea General y las demás leyes y regulaciones que son de aplicación en las coope-

21 Vid. «European Center for Not-for-Profit Law, Legal framework for social Economy and social Enterprise: a comparative report», septiembre, 2012. Disponible en: www.esf.gov.sk. Acceso el 10 de marzo de 2016.

22 Confederación Empresarial Española de la Economía Social (CEPES-2007) Manual para el tutor del Programa RSE.COOP. [on line], disponible en: http://www.cepes.es/listado.cfm?padre=389\&idSeccion=390\&idsec $=s e c \_11$. Acceso el 10 de marzo de 2017.

23 F. Castilla Polo, y D. Gallardo Vázquez, «La revelación social en sociedades cooperativas: una visión comparativa de las herramientas más utilizadas en la actualidad», REVESCO. Revista de Estudios Cooperativos, primer cuatrimestre, vol. 114 (2014): 21.

24 V. gr. Ley No. 95 de fecha 2 de noviembre de 2002, De las Cooperativas de Producción Agropecuaria y de Créditos y Servicios. Gaceta Oficial No. 72 Ordinaria de 29 de noviembre de 2002. 
rativas (disciplina cooperativista); que todos los actos que rigen la vida económica y social de las cooperativas se analizan y deciden en forma democrática por la Asamblea General y la Junta Directiva, en que la minoría acata y se subordina a lo aprobado por la mayoría (decisión colectiva); asimismo que las cooperativas trabajan para lograr la satisfacción racional de las necesidades materiales, sociales, educativas, culturales y espirituales de sus miembros y familiares, entendiéndolo como el principio de bienestar de los cooperativistas y sus familiares, que es una de las vertientes de la responsabilidad social cooperativa; reconoce que las cooperativas se prestan colaboración entre sí sin ánimo de lucro (colaboración entre cooperativas) y que todos sus actos y acciones tienen como fin el interés social.

El legislador del 2002 confundió en un mismo precepto los principios y valores del cooperativismo, incorporando como principio el valor de la solidaridad humana, al reconocer que las cooperativas practican la solidaridad humana con sus miembros, trabajadores y demás personas que habiten en las comunidades donde están enclavadas. No incluyó la educación cooperativa entre los principios, aunque sí reconoce que las cooperativas trabajan en la educación de sus miembros para el cumplimiento de los principios que las rigen, así como en su capacitación técnica y la formación de sus cuadros.

Más recientemente, el artículo 4 del Decreto Ley 305/12 «De las Cooperativas no agropecuarias» 25 , reconoce que I. La incorporación y permanencia de los socios en la cooperativa es libre y voluntaria (VOLUNTARIEDAD), II. Todos los socios trabajan y se prestan ayuda y colaboración entre sí, para alcanzar los objetivos de la cooperativa (COOPERACION Y AYUDA MUTUA), III. LOS actos que rigen la vida económica y social de la cooperativa se analizan y deciden en forma democrática por los socios, que participan en la toma de decisiones

25 Otras normativas se han emitido por el legislador cubano que conforman el marco legal actual de las CNoA: Decreto Ley No. 306/12 que establece el régimen especial de seguridad Social de los Socios de las Cooperativas No Agropecuarias, Decreto No. 309/12, que pone en vigor el reglamento de las cooperativas, Resolución No. 570/2012 del Ministerio de Economía y Planificación que establece el procedimiento de licitación, Resolución No. 427/2012 del Ministerio de Finanzas y Precios que establece los Tributos aplicables a las Cooperativas No Agropecuarias. En su anexo Único establece las normas específicas de contabilidad para las Cooperativas No Agropecuarias y la presentación de sus estados financieros, Resolución No. 426/ 12 que actualiza el Nomenclador y Clasificador del Manual de Normas Cubanas de Información Financiera aplicable a las cooperativas agropecuarias y no agropecuarias. Todas las referencias de publicación en la Gaceta Oficial de la República de Cuba No.No.53 Extraordinaria de 11 de diciembre de 2012. 
con iguales derechos (DECISION COLECTIVA E IGUALDAD DE DERECHOS DE LOS SOCIOS, IV. LaS obligaciones se cubren con los ingresos. Pagados los tributos establecidos, crean los fondos y las utilidades se reparten entre sus socios en proporción a su contribución al trabajo (AUTONOMIA Y SUSTENTABILIDAD ECONOMICA), V. Trabajan por fomentar la cultura cooperativista y por las satisfacciones de las necesidades materiales, de capacitación, sociales, culturales, morales y espirituales de sus socios y familiares (RESPONSABILIDAD SOCIAL) y VI. Las cooperativas se relacionan entre sí y con otras entidades estatales o no, mediante contratos, convenios de colaboración, intercambio de experiencias y otras actividades lícitas (COOPERACION ENTRE (OOPERATIVAS).

Los postulados principistas de las cooperativas cubanas, no están conjugados ni sistematizados como principios propiamente dichos. Mientras que el Decreto Ley No. $142 / 93^{26}$ establece para las UBPC cuatro principios de la actividad y siete características principales de funcionamiento, la Ley No. 95/02 reconoce 10 principios rectores para las CPA y CCS, por su parte el DL 305/12 establece siete principios rectores.

Cualquier cooperativa en el mundo, con independencia del sistema socioeconómico en el cual se desarrolle y la actividad económica a la que se dedique debe cumplir estos principios, no uno o dos de ellos sino todos. Ahora bien, el cumplimiento efectivo de estos principios en el cooperativismo cubano, queda en manos de los propios miembros, no existen mecanismos para su medición y evaluación. En ocasiones se desconoce qué debe inspirar el funcionamiento de una genuina cooperativa y se tergiversa la figura.

En tal sentido, y con el objetivo de evaluar el comportamiento de los principios del cooperativismo en cooperativas del país, se determinó la aplicación de la técnica cualitativa de la entrevista a cooperativistas. Para su aplicación se escogió una muestra en cuatro sectores esenciales: transporte (6 cooperativas) ${ }^{27}$, construcción (32 cooperativas), gastronomía (42 cooperativas), agrícola (15 cooperativas) que operan en ciudad de la Habana; así como, una muestra en el sector agropecuario

26 Decreto Ley No. 142 de fecha 20 de septiembre de 1993, Sobre las Unidades Básicas de Producción Cooperativa. Gaceta Oficial de la República de Cuba No. 6 Extraordinaria de 21 de septiembre de 1993.

27 Más ampliamente sobre las cooperativas del sector del transporte en Cuba véase: Lien Soto Alemán, «Un acercamiento a la implementación de los lineamientos en el sector del transporte cooperativo en Cuba», comp. por Mario Shujman en Las cooperati-

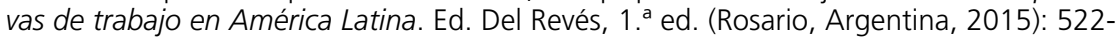
566. 
(12 cooperativas) y 10 en el de la construcción que es el más representativo de la provincia de Matanzas ${ }^{28}$.

Fueron entrevistados socios en 90 CNoA, y en 27 cooperativas agropecuarias (15 CCS y 12 CPA). Se entrevistó a socios en el $100 \%$ de las cooperativas del sector del transporte en ciudad de la Habana, así como en el $100 \%$ de las cooperativas del sector constructivo en la provincia de Matanzas.

El total de CNoA donde se realizaron entrevistas representa el $23 \%$ del total de las constituidas en el país. En el sector agropecuario la cifra fue menor dadas las escasas condiciones de acceso por la distancia.

Entre los entrevistados existen diversas opiniones sobre el proceso de desarrollo de las nuevas cooperativas que se resumen en el aspecto económico. Es el caso de la escasez de recursos y que no poseen un mercado mayorista para la obtención de estos (65\%). En el ámbito de la contratación económica fue reiterativo la mención a que muchas empresas se limitan a no establecer contratos con estas cooperativas por desconocimiento y algunas veces porque creen que no se les permite $(22 \%)^{29}$.

Se reconocen entre los problemas internos, con una visión más social que económica, el hecho de que no existe conciencia de lo que representa ser socios y del significado de una cooperativa por lo que se han dado hechos de muy poca aceptación.

Se enfatizó por varios entrevistados que los beneficios de las cooperativas no son redistribuidos entre sus socios de forma justa y democrática por su trabajo real, sino por conveniencia de los directivos, en

28 Al cierre del año 2015, según datos aportados por el Comité Estatal de Estadísticas en el territorio, se habían constituido en la provincia de Matanzas 14 Cooperativas No Agropecuarias de primer grado, pertenecientes al Ministerio de la Construcción (10), Ministerio del Turismo (2), Ministerio de la Agricultura (1) y Ministerio de Comunicaciones (1). Poco más del $71 \%$ de las CNoA que se encuentran desarrollando su gestión en la provincia pertenecen al Ministerio de la Construcción.

29 Comportamiento errado por parte de las entidades del sector estatal si tenemos en cuenta que el Decreto Ley 304/12 es de vasto alcance para la regulación de la contratación económica entre personas naturales y jurídicas, nacionales y extranjeras, en este último caso siempre que las partes expresamente lo acuerden. Cfr. Decreto Ley No. 304 De la Contratación económica, de fecha 1ro de noviembre de 2012.Gaceta Oficial No.62 Ordinaria de 27 de diciembre de 2012. En adición a esta normativa la Resolución No. 101, de 18 de noviembre de 2011 del Ministro-Presidente del Banco Central de Cuba, que establece las «NORMAS BANCARIAS PARA LOS COBROS Y PAGOS», fue modificada por la Resolución 87/2014 del propio organismo a los efectos de que las cooperativas no agropecuarias puedan recibir pagos en pesos cubanos y en pesos convertibles por parte de las personas jurídicas nacionales, según la moneda en que están autorizadas a operar. 
detrimento de los socios (18\%). No se crea en los asociados una conciencia socialista y de propiedad colectiva. En la mayoría de los casos, un socio fundador (el "gestor») o parte del "comité gestor», financia los gastos de tramitación, constitución y registro de la CNoA, aporta su capital personal para cubrir las aportaciones de los socios y constituir el capital de trabajo inicial, por lo que el resto de los socios no se considera co-propietario, sino empleado de esta.

Se advirtió en otro orden, que no se permite a los asociados una participación activa en la dirección de la cooperativa (31\%), a partir de ello los socios no participan en la toma de decisiones, pues de hecho estas corresponden al presidente y tal vez alguno que otro socio más, por lo que el resto de los asociados tiene una representación formal en la Asamblea General, y carecen de posibilidades de participar en la toma de decisiones democráticas. Se advirtieron actas de la Asamblea General, firmadas solo por el presidente de la CNoA, como si se tratara de un escrito emitido por este, sin que exista constancia de la participación y decisión colegiada de los socios (5\%).

Estas actitudes asumidas en las cooperativas seleccionadas muestran una tergiversación y, en el peor caso, violación del principio cooperativo de control democrático de los asociados, con las consecuencias que de ello se derivan. En el caso de las CNoA fueron en porciento relevante inducidas a su creación por el Estado, con lo que se advierten fallas en el proceso de formación, capacitación y educación de los cooperativistas al momento de adoptar esta nueva forma organizativa.

Es evidente que no se han incorporado como preocupaciones de los cooperativistas la imposibilidad del cumplimiento de su función social. Pareciera lógico en principio, que si presentan problemas con el soporte material para el despliegue de su gestión económica, no pueden acometer e incorporar en la planificación estratégica de su gestión la dimensión social; sin embargo, estas limitaciones de índole económica no deben influir en la visión de los cooperativistas cubanos. Se palpa un evidente desconocimiento, aunque no absolutizamos dado excepciones, de los valores y principios del cooperativismo, lo que transita por un sistema de educación y formación aún deficiente en este sector.

Se aprecia que no contemplan dentro de los grupos de interés a todos aquellos con los cuales debieran sentirse comprometidos socialmente, excluyendo en la mayoría de los casos a los proveedores y no se identifica como necesidad la comunicación con los grupos de interés, fundamentalmente externos, y de los cuales en gran medida puede depender el logro de los objetivos de la organización, lo que impide la posibilidad de generar valores para estos. 
Las acciones sociales acometidas por las cooperativas, a lo interno con los socios y familiares, y a lo externo con la comunidad y los diferentes grupos de interés tienen un carácter aislado.

En cuanto a los principios que informan al sistema cooperativo, los socios que integran las cooperativas entrevistadas no lo conocen, aunque en algunos casos si bien no con el nomen que establece la ley, sí el contenido que informan a estos principios; haciendo gran énfasis cada uno sobre la autonomía, la participación económica de los socios, el control democrático de los miembros y la cooperación entre cooperativas. Ello, como expuso con anterioridad, refleja insuficiencias en los proceso de formación de los miembros de la cooperativa, que guarda relación con sus contenidos así como con la sistematicidad de los mismos.

En relación a los beneficios de los socios todos mencionaron como uno o único, el aumento considerable de «salario» en relación con el salario percibido por los trabajadores estatales, y es muy contradictorio esto si tenemos en cuenta que las cooperativas poseen un objetivo social no lucrativo donde sus miembros perciben anticipos y no salario.

En todas las cooperativas se realiza un contrato al personal, los cuales se encontrarán durante tres meses a prueba y luego la junta de socios decide unánimemente si adquiere la condición de socio. Los horarios de trabajo son fijos, existiendo solo 5 cooperativas que poseen horario de trabajo abierto.

En materia ambiental, no pudieron constatarse acciones que al menos demuestren preocupación por el entorno o los efectos de sus procesos sobre estos, lo que disminuye sus potencialidades para ser consideradas socialmente responsables, pues no basta el crecimiento económico para el logro del desarrollo sustentable si no se tienen en cuenta prácticas de prevención y la protección de los recursos naturales.

Se advierte que no hay preocupación efectiva por imbricarse con la comunidad y que los objetivos estratégicos se guían mayormente por intereses económicos. En la mayoría de los casos los entrevistados confundieron el impulso de acciones sociales responsables hacia las comunidades con el aporte a que vienen obligadas por ley ${ }^{30}$.

30 Ley No. 113 de fecha 23 de julio de 2012, Del sistema Tributario. Gaceta Oficial No. 53 Extraordinaria de 21 de noviembre de 2012.

«Art. 305. - Se establece una Contribución para el desarrollo sostenible de los municipios, que grava los ingresos por la comercialización de bienes o prestación de servicios, que obtengan las empresas, sociedades mercantiles y cooperativas, por sí mismas y por sus establecimientos en cada territorio.

Art. 306.-Son sujetos de esta Contribución, las empresas, las sociedades mercantiles de capital totalmente cubano y las cooperativas, por sus establecimientos, sin perjuicio del municipio en que estén enclavados». 
En igual orden, salvo 3 entrevistados el resto demostró confusión entre los conceptos de BSCoop y balance económico, respondiendo por este último al preguntarle por el primero.

En el caso de los asociados entrevistados en las cooperativas del sector agropecuario, un número significativo ( $80 \%$ ) demostró tener un buen conocimiento de sus derechos como miembros de la cooperativa, sin embargo, en relación a la satisfacción interna como asociados no se obtuvo igual reconocimiento. No se perciben acciones sociales a lo interno de la cooperativa que empodere a los asociados.

A pesar de los problemas descritos, es indudable que las cooperativas del sector no agropecuario constituyen una nueva forma de gestión que genera nuevas opciones de trabajo al tiempo que cubre servicios de insuficiente cobertura por las empresas estatales, o que estas realizaban de forma deficiente, ocurriendo en la práctica que el servicio de las cooperativas posee mayor calidad que el estatal. Además, en su seno se fomenta el sentido de pertenencia, preservándose los medios de trabajo y tratando en todo momento de hacer que la actividad económica que desarrollan sea rentable a corto y largo plazo; incentivando la competencia con otras entidades que prestan igual servicio. Por su parte las potencialidades de las cooperativas del sector agropecuario se mantienen de acuerdo a las proyecciones que históricamente se han visionado para estas formas organizativas, los que en el momento actual se acrecientan. Esto en el aspecto económico. El que nos motiva con la presente investigación: el aspecto social de la gestión de las cooperativas, amerita otros comentarios.

Los estatutos revisados mostraron que para dar cumplimiento al objeto social de las CNoA se establecían algunas actividades de apoyo, con evidente fin social (25\%), a lo interno de la cooperativa, pueden resumirse en las siguientes:

- Brindar a los socios servicios gastronómicos, constructivos y otros relacionados con adquirir bienes de consumo, para elevar la calidad de vida de los mismos y sus familias.

- Promover entre sus asociados el conocimiento y la educación de los principios y métodos cooperativos y difundirlos por todos los medios a su alcance.

- Implementar un programa de desarrollo que permita aumentar el nivel técnico y profesional de los cooperativistas en materia de construcción.

A pesar de ello cuando se preguntaba cómo operaba en la práctica la proyección contenida en los estatutos manifestaron que ello tenía lugar de forma eventual, en la mayoría de los casos no incorporada a la 
proyección estratégica de la cooperativa, con lo cual resulta contradictorio lo expresado en estatutos y la viabilidad de esto.

En relación a las cooperativas del sector agropecuario aun cuando no se pudo tener acceso a los estatutos, en las entrevistas formuladas a los asociados se pudo conocer que aunque se prevén acciones a lo interno de las cooperativas encaminadas a la satisfacción personal de los asociados y sus familiares, las limitaciones materiales restringen el nivel de satisfacción.

\section{Experiencias de Balance Social Cooperativo en Cuba}

En los últimos años se han incrementado los estudios en el país sobre los diferentes modelos de balance social aplicados a las cooperativas, en La Habana, Pinar del Río y Villa Clara. Aunque se han centrado en el sector agropecuario, arrojan elementos importantes a analizar en la construcción de un modelo de BSCoop integral aplicable a todas las cooperativas. El primero de ellos fue desarrollado por el Centro de Estudios de Desarrollo Cooperativo y Comunitario (CEDECOM) de la Universidad de Pinar del Río. Al frente del equipo de trabajo se encontraba el Dr. Juan Luis Alfonso Alemán, los que presentaron un modelo de gestión social en la Cooperativa de Créditos y Servicios «Camilo Cienfuegos» ${ }^{31}$.

En este se explica que el modelo de evaluación del impacto social debe constituirse, de manera general, conteniendo dos bloques o subsistemas analíticos - informativos: 1) Referido a la evaluación del grado de cumplimiento de los principios del cooperativismo (Subsistema I) y 2). Referido a la construcción y evaluación del Balance Social (Subsistema II). Ambos subsistemas deberán definirse atendiendo a las áreas internas y externas de actuación de las cooperativas. De esta manera, el proceso de autoevaluación de la acción social permite incorporar, a la dinámica ya habitual en muchas cooperativas en su desempeño económico; su acción y diagnóstico de su responsabilidad social (...). Según la metodología diseñada para la evaluación de los principios cooperativos, en el marco del Proyecto de Balance Social de 1997, auspiciado por la Organización Internacional del Trabajo (OIT) y por la ACl; y a partir de los subsistemas que permiten valorar integralmente la acción social de las cooperativas, se consideró incluir el sistema de indicadores

31 Juan Luis Alfonso Alemán et al, «Responsabilidad y balance social en las empresas cooperativas», en Revista de Ciencias Sociales, Vol. 14, No. 1, (2008): 9-19. 
generales que contiene ese modelo, a partir de la operacionalización de los principios cooperativos ${ }^{32}$.

El modelo fue extendido posteriormente, en el año 2009, a la CPA «14 de junio», en la propia provincia de Pinar del Rio. Tuvo como principal objetivo diseñar un Sistema de Contabilidad del Balance Social para las Empresas Cooperativas que permita registrar, medir y evaluar su desempeño o acción social. Para el estudio del modelo fueron analizados los indicadores sociales más representativos: Solvencia Social, Rentabilidad social y Liquidez Social, en correspondencia a las técnicas utilizadas en la dinámica económica y financiera, pero ajustadas a la especificidad de la acción sociaß33

Dada la complejidad del modelo, este fue perfeccionado con posterioridad, en función de lograr la motivación de las entidades cooperativas en su aplicación efectiva. La implementación del modelo perfeccionado de gestión social se realizó durante el período 2011 a primer semestre de 2012 y abarcó un total de 6 formas organizacionales cooperativas (también del sector agropecuario) en las que se logró demostrar la validez y utilidad de dicho proceso de perfeccionamiento. Entre los impactos más significados fueron señalados sucintamente los siguientes:

- Elevación de los niveles de eficiencia y eficacia socioeconómicas al disminuir los costos de producción y fortalecimiento del Control Interno, a partir del perfeccionamiento de la contabilidad a todos los niveles en el uso de los recursos.

- Elevación del sentido de pertenencia de los trabajadores y asociados a las cooperativas, que se refleja en la no ocurrencia de bajas y en el incremento de la productividad del trabajo.

- Incremento en la incorporación de la mujer al trabajo de las cooperativas por la construcción de jardines de la infancia, garantizarse el aseguramiento material y alimentario de los mismos, y lograr la asesoría y preparación del personal de estas instalaciones por las direcciones municipales de educación de los territorios.

- Capacitación técnica y ética de los recursos humanos de las empresas cooperativas, tanto de los cuadros, como de los asociados.

32 Juan Luis Alfonso Alemán et al, «Responsabilidad y balance social», 11.

33 Alain Hernández Santoyo et al, «La Gestión y el Balance Social en la Empresa Cooperativa Cubana. Caso de Estudio: CPA 14 de junio», en Revista Contabilidad y Auditoría, No. 29, (2009): 65. 
— Fortalecimiento del trabajo del gobierno en las comunidades: Delegados del Poder Popular y Presidentes de Consejos Populares, así como la consolidación de los procesos de toma de decisiones sobre la base de la participación de los ciudadanos ${ }^{34}$.

El segundo modelo fue desarrollado por el Instituto de Ciencia Animal (ICA)-Facultad Latinoamericana de Ciencias Sociales, de la Universidad de La Habana (FLACSO)-Programa Conjunto de Naciones Unidas $(P C)^{35}$, mediante la tesis de maestría de la Ing. Cira María Mena Lazo, este modelo profundiza en el área de intervención equidad de género y en cuestiones medioambientales, incorpora la evaluación de los principios cooperativos e indicadores económicos y productivos, así como establece una guía de pasos para la consumación del modelo. Fue implementado en la UBPC «Las Cadenas» del municipio La Palma, en la provincia de Pinar del Rio con un alto impacto en los resultados socioeconómicos y medioambientales. Según MENA LAZO se trata de un modelo representado por un sistema de variables e indicadores, que facilita el cálculo para la evaluación. Está dividido en áreas de intervención donde concurren indicadores cualitativos y cuantitativos, internos y externos. El modelo de Balance Social propuesto, interviene en el área de "Protección al Medio Ambiente», con el objetivo de ayudar a la toma de decisiones en las cooperativas, modificar actitudes y prácticas indolentes, respecto a los principales recursos naturales como la tierra, agua, aire, árboles ${ }^{36}$.

Por su parte, en el sector no agropecuario la Cooperativa Reconstructora de Vehículos de La Habana ha desarrollado prácticas de contenido social, sin llegar a desarrollar ninguno de los modelos precedentes. La cooperativa decidió crear un fondo de entre 18 y 20 mil CUC para préstamos sin intereses a los socios ante determinadas eventualidades, o para premiar a los trabajadores más destacados. Para validar el principio cooperativista del aporte social la cooperativa atiende con precios moderados a los socios, propietarios de autos, jubilados o no, que no poseen la capacidad adquisitiva para contratar esos servicios. Apadrinó el Asilo Juan Lefont, del Municipio Cerro realizando reparaciones de neveras y camas, la entrega de una bomba de agua y un

34 Más ampliamente véase: Juan Luis Alfonso Alemán y Claudio Alberto Rivera Rodríguez, «Perfeccionamiento del Modelo de Gestión Social en las empresas cooperativas de la provincia de Pinar del Río. Resultados e Impactos», Revista Avances, Vol. 15, No. 1 enero-marzo (2013): 47.

35 Para más ampliación sobre el modelo véase: Cira Mena et al, Modelo de Balance Social Cooperativo ICA-FLACSO-PC. Compendio para la implantación. La Habana, julio 2014.

36 Cira Mena, et al., «Modelo de Balance...», 9. 
freezer de almacenamiento. Asimismo reparó 138 equipos, a precio acorde a las posibilidades de pago de las personas que los requirieron. Una de las líneas de trabajo incorporada está vinculada con la recuperación de ambulancias y en este ámbito se reconstruyeron vehículos a un costo promedio de 3.833 CUC, contribuyendo de esta forma con el Sistema de Salud Pública del país ${ }^{37}$.

EI BSCoop de esta entidad fue realizado por primera vez en 2014 por la Comisión de Arbitraje y Atención a los Socios. Aunque realmente constituye un informe contentivo de las principales acciones acometidas por la cooperativa en el ámbito social, no trabaja con indicadores sistematizados, y su registro en la contabilidad es el tradicional. A criterio de esta autora, a pesar del salto cualitativo que representan estas aptitudes, no dejan de ser eso, y el reto continúa siendo que sea incorporado a la estrategia organizacional de las cooperativas en base a un modelo de BSCoop que establezca los indicadores fundamentales susceptibles de evaluación por auditores externos a la cooperativa.

\section{A favor de un marco jurídico para la utilización del balance social cooperativo}

La base voluntaria sobre la cual se sustenta el principio de responsabilidad social en las cooperativas plantea múltiples posturas con relación a su contenido, verificación y obligatoriedad. Con relación al primer aspecto, la información contenida es tremendamente heterogénea consecuencia de la discrecionalidad que tiene cada entidad. Esta flexibilidad que permite la adaptación de cada organización a su actividad y a los objetivos fijados de cara a sus interlocutores, puede plantear problemas de comparabilidad entre las cooperativas. En Cuba estas organizaciones han venido desarrollando acciones socialmente responsables, pero como ha quedado demostrado con anterioridad, son eventos aislados, carentes de sistematicidad, y no están incorporados a la gestión económica de estas. Lógicamente, depende ello del escenario económico en el cual se desarrollan las cooperativas, los beneficios y los excedentes percibidos al finalizar el ejercicio fiscal son lo que motiva esta actuación.

37 Más ampliamente véase: Mirta Vuotto y Verbeke, G., «Las cooperativas no agropecuarias en Cuba en el contexto de transformación de la Cuba de hoy. Desafíos actuales». (Conferencia XII Congreso Nacional de estudios del trabajo. Grupo Temático N. ${ }^{\circ}$ 11: Trabajo y autogestión en las organizaciones de la economía Social (Conferencia, Buenos Aires, Argentina, agosto 2015). 
El aspecto económico es esencial, una cooperativa ineficiente, cualquiera sea el sector en que se desarrolla, no puede asumir compromisos sociales, por más que quiera. Necesita del soporte material, al cual se hizo referencia de forma precedente aludiendo a Alfonso Alemán.

La actualización del modelo económico cubano, con sus multicausalidades le otorga un papel esencial y complementario del sector empresarial estatal38; sin embargo este encargo "económico» no puede desvirtuar las esencias de la figura, ya que su existencia como forma especial de asociación de personas con un objetivo común, las obliga a gestionar adecuadamente todas sus dimensiones y no únicamente las económicas.

Su misión social les viene por mandato del artículo 4 del Decreto Ley $305 / 12$ que refrenda la responsabilidad social entre los principios identitarios que las impulsan, a lo interno con los socios y trabajadores, y a lo externo con los clientes, proveedores, gobiernos locales y comunidades.

El BSCoop como herramienta de evaluación del cumplimiento de los principios cooperativos deberá desarrollarse a partir de un conjunto de presupuestos teóricos-jurídicos derivados de la propia esencia y naturaleza cooperativas, y concretados en las siguientes líneas, los que sistematizados deberán considerarse en la futura Ley General de Cooperativas en Cuba:

- Balance Social Cooperativo:

Es una herramienta de gestión empresarial, contenido en un informe de alcance público, que permite la evaluación interna y externa del cumplimiento de la responsabilidad social de la cooperativa en un periodo determinado, mediante el uso de indicadores sociales, con el objetivo de lograr una evaluación integral de los procesos.

- El carácter público:

Posibilita comunicar a los grupos de interés los resultados de la gestión social de la cooperativa. Por ello debe considerarse una herramienta de comunicación de la responsabilidad social cooperativa. Permitiría en el contexto cubano la comparación por las entidades verificadoras y auditores del grado de cumplimiento de los principios cooperativos, estimulando las mejores cooperativas y desenmascarando las falsas que puedan crearse (carácter externo) y a las propias cooperativas rediseñar sus estrategias, establecer comparaciones con etapas precedentes, en fin, autoevaluarse (carácter interno). El espacio temporal coinci-

38 Numeral 2 de los Lineamientos, p 10. 
diría con el espacio del balance financiero, año fiscal, y respondería esta herramienta a estrategias previamente diseñadas y acordadas por todos los socios.

La publicidad del BSCoop tendría lugar a través del registro mercantil, sin que dejemos de significar que en el contexto cubano se trata de una publicidad abierta decretada en norma pero cerrada en la práctica, visión que deberá corregirse para lograr el verdadero propósito del BSCoop ${ }^{39}$.

- El carácter obligatorio:

De los análisis presentados en las líneas precedentes, se considera que la información social contenida en el BSCoop debe tener carácter obligatorio, cualquiera sea el sector en que se desarrolla la cooperativa así como el número total de socios.

La regulación de este aspecto posibilita una evaluación eficiente del cumplimiento de los principios del cooperativismo, permite valorar cuantitativa y cualitativamente el impacto de la gestión social cooperativa a lo interno, con los socios, y a lo externo con las comunidades, los clientes, las instituciones del Estado y la sociedad en general. La operacionalización de estos indicadores debe establecerse sobre la base de criterios diagnóstico, algunos pueden establecerse de forma autónoma por las cooperativas, otros por el contrario deben estar legalmente establecidos. Este sistema de indicadores puede ser diferente atendiendo al tipo de cooperativa, los indicadores de las cooperativas del sector agropecuario pueden ser diferentes a las del sector no agropecuario, de ahí su carácter flexible.

La determinación de la metodología para la elaboración del BSCoop, las áreas de intervención y las variables e indicadores sociales a considerar en su elaboración, rebasa los propósitos de la presente investigación, el tema en cuestión demanda investigaciones interdisciplinarias; en tal sentido economistas y sociólogos deberán involucrarse en su diseño, los primeros para la elaboración de una metodología del BSCoop, los segundos para la determinación de las variables e indicadores sociales.

39 Acrece la problemática el hecho de que la norma legal que regula el Registro Mercantil (Resolución 230/02 del MINJUS) establece que el Reglamento se aplicará con carácter provisional por el transcurso de un año, sin que se haya emitido la nueva normativa y aplicándose en la práctica su contenido sin el debido fundamento legal. Vid. Resolución No. 230/02 «Reglamento del Registro Mercantil» de 29 de octubre de 2002. Gaceta Oficial, Edición Ordinaria No. 58 de fecha 4 de noviembre de 2002. 
- Representa información cuantificable:

En otro orden, la distribución de excedentes y el reparto de los beneficios parten de la autonomía de la voluntad de los socios, plasmado en sus estatutos, pero en función del compromiso social de estas entidades debe incorporarse a la contabilidad como parte de la sistemática del proceso. En otras palabras, la contabilidad debe contribuir a informar sobre la forma en que se ha generado el valor agregado y la forma en que se ha distribuido, poniendo énfasis en el aspecto social. Y es la verificación de la información social contenida en la contabilidad realizada por un profesional externo lo que la hace confiable, en base al principio de transparencia de la contabilidad. De ahí la importancia de la auditoria en este aspecto.

- Es instrumento de control (interno y externo):

No basta con la incorporación de criterios éticos a la estrategia de gestión de la cooperativa, es importante hacerlo en base a indicadores que permitan evaluar y comprobar que efectivamente se están cumpliendo. Ya apuntábamos de forma precedente que los indicadores sociales permiten establecer parámetros de comparabilidad entre las cooperativas al momento de la evaluación de la gestión.

El BSCoop regulado en una futura normativa con carácter de Ley y aplicable a todas las cooperativas debe contener la identificación del organismo que se encargue de verificar y evaluar su contenido, para darle el sentido coactivo propio de la Ley ${ }^{40}$.

- El papel del Estado. Regulación vs fomento:

Otra premisa importante para la gestión social, está dada por el reconocimiento y apoyo del Estado a la acción social directa de las cooperativas. El Estado puede promover prácticas socialmente responsables mediante exenciones fiscales y bonificaciones para aquellas cooperativas que obtienen resultados satisfactorios, así como el financiamiento a programas de desarrollo de estas prácticas. No debe pensarse en una responsabilidad social limitada únicamente a quienes deben llevarla a cabo, el Estado tiene un rol esencial y en este sentido puede establecer un marco referencial mínimo para la promoción y estímulo de acciones responsables. Las cooperativas del sector agropecuario cuentan hoy con la Asociación Nacional de Agricultores Peque-

40 Vid Infra, «Es información auditable». 
ños, sus homólogas del sector no agropecuario son atendidas con carácter transitorio por la Comisión permanente para la implementación de los Lineamientos del Partido. Con lo cual el escenario inmediato demanda de un organismo de carácter nacional, con ramificaciones a nivel local que promueva, represente y supervise a todas las cooperativas en el país. Aún más allá, en la construcción del modelo cooperativo al cual debe aspirar el país, debe pensarse en la creación de un órgano de fomento y supervisión para todo el sector cooperativo ${ }^{41}$.

En general, las medidas de fomento hacia el sector cooperativo, armonizarían con la Recomendación sobre la promoción de las Cooperativas 2002 de la OIT (R 193). En tal sentido los Estados deben adoptar medidas tendentes a promover el potencial de las cooperativas con el fin de ayudarlas a ellas y a sus asociados a crear y desarrollar actividades generadoras de ingresos y empleo decente, fomentar el conocimiento de los valores y principios del cooperativismo, así como de sus ventajas y beneficios mediante la educación y la formación ${ }^{42}$.

En otro orden, el reconocimiento y apoyo del Estado a la acción social directa de las cooperativas deberá basarse en un redimensionamiento de sus relaciones con el cooperativismo, a partir de la adopción de instrumentos legales que despojen a la gestión social de su carácter voluntario u opcional. Se trata de la regulación con carácter obligatorio del uso del balance social por las cooperativas.

- Es información auditable:

El informe social acerca del desempeño de la cooperativa, con la evaluación de sus indicadores sociales, así como el análisis de su Balance Social, es auditable.

En Cuba la Contraloría General de la República tiene por mandato según el artículo 1.5 de la Ley $107 / 09^{43}$ ejercer sus funcio-

41 No es objetivo de este trabajo profundizar en la propuesta de organismo, sobre todo en un contexto actual donde se aboga por la concentración de ministerios, baste solo insistir en su necesidad.

42 Conferencia General de la Organización Internacional del Trabajo: Convocada en Ginebra por el Consejo de Administración de la OIT, 3 de junio de 2002, disponible en: www.ilo.org/dyn/normlex/es/f?p=1000:12100:0::NO::P12100. Acceso el 15 de septiembre de 2016.

43 Ley No. 107 De la Contraloría General de la República, de fecha 1 ro de agosto de 2009. Gaceta Oficial de la República de Cuba No. 29 Extraordinaria de 14 de agosto de 2009. 
nes en los órganos, organismos, organizaciones y entidades que reciban recursos del Estado para su gestión o estén sujetos a una obligación tributaria generada en el país, en la forma y oportunidad que la Ley establece, en este último caso estarían incluidas las cooperativas.

La futura normativa debe establecer que junto a las acciones de supervisión y auditoría del balance económico-financiero tradicional se supervise y audite el balance social.

Deberá incluirse en el futuro Reglamento General para las cooperativas, las sanciones de carácter administrativo que se aplicarán a las cooperativas que no cumplan con el reporte del BSCoop o que tengan un desempeño social no satisfactorio.

\section{Conclusiones}

Teniendo en cuenta los objetivos trazados para llevar a cabo la presente investigación, señalamos las conclusiones siguientes:

Primera. Las cooperativas, aunque son entidades sin fines de lucro, presentan condicionantes económicas para su propia sustentabilidad. Por ello, la correcta articulación del análisis económico de la gestión de estos sujetos debe incorporar de manera expresa la dimensión social a fin de que se logre una evaluación integral de los procesos.

Segunda. El BSCoop mide la Responsabilidad Social Cooperativa en relación con el cumplimiento de los principios cooperativos que se sostienen en un basamento de valores. Con este fundamento el BSCoop aparece como una herramienta de la gestión socioeconómica que les facilita a las cooperativas medirse y rendir cuentas a los/as asociados/as y a todos los demás grupos de interés que están impactados por su accionar, así como del cumplimiento de sus valores y principios, y del impacto social de la organización en su comunidad.

Tercera. La valoración en la práctica del comportamiento de los principios del cooperativismo en la muestra de cooperativas seleccionadas en el país, evidenció que las acciones sociales desarrolladas tienen un carácter aislado y asistemático, sin que se incorporen a la gestión económica de las cooperativas.

Cuarta. La necesidad de disponer el uso del BSCoop con carácter obligatorio como herramienta de evaluación de los principios cooperativos en las cooperativas cubanas, surge por la confluencia de dos pilares fundamentales: de una parte la existencia de instrumentos de gestión económica, que en muchos casos presentan análisis limitados y alejados de la naturaleza de la identidad cooperativa, por lo que resul- 
tan incompletos; y de otra, la ausencia de indicadores sociales que limita la evaluación del impacto social de la cooperativa.

Quinta. La implementación del BSCoop proporciona como beneficios: la autoevaluación de la cooperativa, la información y rendición de cuentas a los miembros y a la comunidad sobre el impacto social de las actividades de la cooperativa. Posibilita además, para las autoridades controladoras y evaluadores, la presentación de resultados comparables en relación al grado de cumplimiento de los principios cooperativos y la consecuente adopción en el orden administrativo de las medidas que correspondan. Legitimaria el control popular sobre la gestión socioeconómica de la cooperativa.

Sexta. El BSCoop es un complemento del balance económico tradicional y constituye una herramienta de apalancamiento de la responsabilidad social de las cooperativas. En tal sentido, para que las cooperativas cubanas concreten sus potencialidades de responsabilidad social y se consoliden como un movimiento unitario, es necesario crear un entorno regulatorio e institucional propicio, que implica la emisión de una Ley general de cooperativas que ordene y sistematice los principios generales del cooperativismo en Cuba y establezca con carácter obligatorio el uso del BSCoop.

\section{Bibliografía}

ALBUQUERQUE LLORENS, Francisco, «Desarrollo económico local y descentralización en América Latina», en Revista de la CEPAL, No. 82 (2004): 21-52.

ALFONSO ALEMÁN, Juan Luis, RIVERA RODRÍGUEZ, Claudio Alberto, LABRADOR MACHÍN, Odalys, «Responsabilidad y balance social en las empresas cooperativas», en Revista de Ciencias Sociales, Vol. 14, No. 1 (2008): 09-19.

- , «Responsabilidad social empresarial. Sus particularidades en las empresas cooperativas», en Revista Avances, Vol. 11, No. 1, (2009), [on line], disponible en: http://www.ciget.pinar.cu/Revista/No.2009-1/articulos/Responsabilidad_social.pdf. Acceso el 10 de octubre de 2016.

ALFONSO ALEMÁN, Juan Luis y Claudio Alberto RIVERA RODRÍGUEZ, "Perfeccionamiento del Modelo de Gestión Social en las empresas cooperativas de la provincia de Pinar del Río. Resultados e Impactos», Revista Avances, Vol. 15, No.1 enero-marzo, (2013): 40-53.

BELHOUARI, Adil, Inmaculada BUENDÍA, Marie Josée LAPOINTE y Benoît TREMBLAY, «La responsabilidad social de las empresas: ¿Un nuevo valor para las cooperativas?», en Revista CIRIEC ESPAÑA de Economía pública, social y cooperativa, No. 53, (2005): 191-211.

CAROSINI, Leticia, Visión panorámica del sector cooperativo en Paraguay. Una importante contribución al desarrollo nacional, Oficina de la OIT para los Países Andinos, La Paz, 2012. [on line] disponible en: 
http://www.ilo.org/santiago/publicaciones/WCMS_191115/lang--es/index. htm. Acceso el 10 de agosto de 2016.

CARRERAS, LI. y BASTIDA, R., «Estudio sobre la rendición de cuentas en materia de responsabilidad social: el balance social», Revista CIRIEC ESPAÑA de Economía pública, social y cooperativa, No. 84, (2015): 251-277.

CASTILLA POLO, F. y D. GALLARDO VÁZQUEZ, «La revelación social en sociedades cooperativas: una visión comparativa de las herramientas más utilizadas en la actualidad», REVESCO. Revista de Estudios Cooperativos, primer cuatrimestre, vol. 114, (2014): 07-34.

CRACOGNA, Dante, "El Balance Social de la Empresa», en Revista Administración de Empresa, No. 124, (1980): 36-52.

Diccionario de la Lengua Española, 23 a. Ed, 2014. [on line], disponible en: http://www.rae.es/diccionario-de-la-lengua-espanola/la-23a-edicion-2014. Acceso el 21 de enero de 2016.

GARCÍA MULLER, Alberto, Derecho Cooperativo y de la Economía Social y Solidaria, Asociación Iberoamericana de Derecho Cooperativo, Mutual y de la Economía Social y Solidaria, Mérida, 2015, [on line], disponible en: www. oescj.org.ec/pdf/bibliotecal.../Derecho-Cooperativo-Economia-social-solidaria. Acceso el 22 de abril de 2016.

HERNÁNDEZ SANTOYO, Alain, Víctor E. PÉREZ LEÓN y Juan Luis ALFONSO ALEMÁN, «La Gestión y el Balance Social en la Empresa Cooperativa Cubana. Caso de Estudio: CPA 14 de junio», en Revista Contabilidad y Auditoría, No. 29, (2009): 63-77.

MENA LAZO, C., O. LLÁNEZ GUERRA, Reynaldo JIMÉNEZ GUETHÓN y O. ARJONA VÁZQUEZ, Modelo de Balance Social Cooperativo ICA-FLACSO-PC. Compendio para la implantación. La Habana, Cuba, 2014.

MUGARRA ELORRIAGA, Aitziber, «Proyecto de Balance de Identidad Cooperativa», en Anuario de Estudios Cooperativos, (Universidad de Deusto, Bilbao, 1999): 303-390.

- , "Responsabilidad y balance social hoy en día: un reto para las cooperativas». CIRIEC, Revista Jurídica de Economía Social y Cooperativa, No. 39, (2001): 25-50.

- "Modelos de Balance Social: Criterios clasificatorios», Instituto de Estudios Cooperativos COLECCION DE CASOS, (Universidad de Deusto, Bilbao, 2009): 1-28

NOVA GONZÁLEZ, Armando, Las cooperativas agropecuarias en Cuba: 1959presente, comp. por Camila Piñeiro Harnecker, Cooperativas y Socialismo: Una mirada desde Cuba, Editorial Caminos, La Habana 2011.

SAN BARTOLOME, Juan Carlos, GONZÁLEZ, L., Balance Social Cooperativo: Una construcción en construcción, Ediciones Red Gráfica, Buenos Aires, 2008.

SAN BARTOLOME, Juan Carlos, Balance social cooperativo: una responsabilidad intrínseca de la empresa cooperativa. Foro Balance Social Cooperativo y Gobernabilidad. XVII Conferencia Regional de ACI AMERICAS. Conferencia pronunciada en Buenos Aires, Argentina el 22 de noviembre de 2010.

SOTO ALEMÁN, Lien, «Un acercamiento a la implementación de los lineamientos en el sector del transporte cooperativo en Cuba», comp. por Mario 
Shujman en Las cooperativas de trabajo en América Latina. Ed. Del Revés,

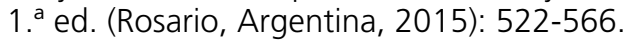

-, La Responsabilidad Social Empresarial en Cuba: Una visión desde el conocimiento jurídico en pos del desarrollo local», en Revista Jurídica DEREITO, Universidad Santiago de Compostela, España, Vol.25, No.1, (2016): 101115.

VUOTTO MIRTA y VERBEKE, G., «Las cooperativas no agropecuarias en Cuba en el contexto de transformación de la Cuba de hoy. Desafíos actuales». Conferencia XII Congreso Nacional de estudios del trabajo. Grupo Temático N. ${ }^{\circ}$ 11: Trabajo y autogestión en las organizaciones de la economía Social. Conferencia prounciada en Buenos Aires, Argentina en agosto de 2015.

\section{Documentos}

Alianza Cooperativa Internacional, Plan para una década cooperativa, Visión 2020, 2013, [on line], disponible en: http://www.aciamericas.coop/Planpara-una-Decada-Cooperativa. Acceso el 20 de octubre de 2016.

Confederación Empresarial Española de la Economía Social (CEPES) Manual para el tutor del Programa RSE.COOP. 2007, [on line], disponible en: http://www.cepes.es/listado.cfm?padre=389\&idSeccion=390\&idsec=sec_1 1. Acceso el 10 de marzo de 2017.

European Center for Not-for-Profit Law, «Legal framework for social Economy and social Enterprise: a comparative report», septiembre, 2012. [on line], disponible en: www.esf.gov.sk. Acceso el 10 de octubre de 2016.

Ley Marco para las cooperativas de América Latina/ Alianza Cooperativa Internacional para las Américas. - 1a ed.- San José, Costa Rica: Alianza Cooperativa Internacional para las Américas, 2009.

Organización Internacional del Trabajo, La Responsabilidad social corporativa en Santander Central Hispano, Base de datos fuente académica, 2006, [on line], disponible en: wWw.ilo.org/empent/Publications/WCMS_142694/ lang--es/. Acceso el 29 de noviembre de 2015.

Organización Internacional del Trabajo, Conferencia General convocada en Ginebra por el Consejo de Administración de la OIT, en su 90. a reunión, Ginebra, 2002, [on line], disponible en: www.ilo.org/dyn/norm/ex/es/ $f ? p=1000: 12100: 0:$ : Acceso el16 de febrero de 2016.

Organización Internacional del trabajo; «Estrategia global en materia de seguridad y salud en el trabajo». Conclusiones adoptadas por la Conferencia Internacional del Trabajo en su 91. a reunión. Ginebra, 2003. [on line], disponible en: www.ilo.org/public/spanish/standards/relm/ilc/ilc91/pdf/rep-vi. pdf. Acceso el 21 de diciembre de 2015.

Partido Comunista de Cuba, VII Congreso: Actualización de los Lineamientos de la Política Económica y Social del Partido y la Revolución para el período 2016-2021, abril de 2016, Disponible en: http://www.cubadebate.cu/noticias/descargue-en-cubadebate-los-lineamientos-de-la-politica-economicay-social-pdf/. Acceso el 12 de enero 2017. 


\section{Legislación}

Ley No. 95, De las Cooperativas de Producción Agropecuaria y de Créditos y Servicios, de fecha 2 de noviembre de 2002.Gaceta Oficial No. 72 Ordinaria de 29 de noviembre de 2002 .

Ley No. 107 de fecha 1ro de agosto de 2009, De la Contraloría General de la República. Gaceta Oficial de la República de Cuba No. 29 Extraordinaria de 14 de agosto de 2009.

Ley No. 113 de fecha 23 de julio de 2012, Del sistema Tributario. Gaceta Oficial No. 53 Extraordinaria de 21 de noviembre de 2012.

Ley Orgánica de la Economía Popular y Solidaria del Sistema Financiero Popular y Solidario de Ecuador, de 28 de abril de 2011. Registro Oficial 444 de 10 de mayo de 2011. Disponible en: http://www.oficial.ec/decreto-679-reformese-reglamento-general-ley-organica-economia-popular-solidaria. Acceso el 25 de diciembre de 2016.

Decreto Ley No. 142 Sobre las Unidades Básicas de Producción Cooperativa, de fecha 20 de septiembre de 1993. Gaceta Oficial de la República de Cuba No. 6 Extraordinaria de 21 de septiembre de 1993.

Decreto Ley No. 304 De la Contratación económica, de fecha 1ro de noviembre de 2012. Gaceta Oficial No.62 Ordinaria de 27 de diciembre de 2012.

Decreto Ley No. 305 De las Cooperativas no Agropecuarias, de fecha 15 de noviembre de 2012. Gaceta Oficial No.53 Extraordinaria de 11 de diciembre de 2012.

Decreto Ley No. 306/12 Del régimen especial de seguridad Social de los Socios de las Cooperativas No Agropecuarias, de fecha 28 de noviembre de 2012. Gaceta Oficial No. 53 Extraordinaria de 11 de diciembre de 2012.

Decreto No. 309 Reglamento de las Cooperativas no Agropecuarias de primer grado, de fecha 28 de noviembre de 2012. Gaceta Oficial No. 53 Extraordinaria de 11 de diciembre de 2012.

Resolución No. 230/02 Reglamento del Registro Mercantil, de 29 de octubre de 2002. Gaceta Oficial, Edición Ordinaria No. 58 de fecha 4 de noviembre de 2002.

Resolución No. 101, de 18 de noviembre de 2011 del Ministro-Presidente del Banco Central de Cuba, que establece las «NORMAS BANCARIAS PARA LOS COBROS Y PAGOS». Gaceta Oficial de la República de Cuba No. 40 Extraordinaria de 21 de noviembre de 2011.

Resolución No. 574 de fecha 13 de agosto de 2012 del Ministro de la Agricultura, Reglamento General de las Unidades Básicas de Producción Cooperativa. Gaceta Oficial de la República de Cuba No. 37 Extraordinaria de 11 de septiembre de 2012.

Resolución No. 427/12 de fecha 4 de diciembre de 2012 de la Ministra de Finanzas y Precios, Norma específica de Contabilidad No. 7 para cooperativas no agropecuarias. Gaceta Oficial de la República de Cuba No. 53 Extraordinaria de 11 de diciembre de 2012. 
Resolución No. 570/2012 del Ministro de Economía y Planificación, establece el procedimiento de licitación. Gaceta Oficial de la República de Cuba No. 53 Extraordinaria de 11 de diciembre de 2012.

Resolución 87/2014 del BCC. Publicada en la Gaceta Oficial No. 42 Extraordinaria de 2011. Gaceta Oficial No. 40. Extraordinaria de 30 de septiembre de 2014.

Resolución No. 4109 de fecha 30 de enero de 2009 del Presidente del Instituto Nacional de Cooperativismo de Paraguay, "Balance social cooperativo». Disponible en: http://www.incoop.gov.py/v1/?s=4109. Acceso el 22 de enero de 2016. 


\section{Derechos de autor}

El Boletín de la Asociación Internacional de Derecho Cooperativo es una revista de acceso abierto lo que significa que es de libre acceso en su integridad inmediatamente después de la publicación de cada número. Se permite su lectura, la búsqueda, descarga, distribución y reutilización legal en cualquier tipo de soporte sólo para fines no comerciales y según lo previsto por la ley; sin la previa autorización de la Editorial (Universidad de Deusto) o el autor, siempre que la obra original sea debidamente citada (número, año, páginas y DOI si procede) y cualquier cambio en el original esté claramente indicado.

\section{Copyright}

The International Association of Cooperative Law Journal is an Open Access journal which means that it is free for full and immediate access, reading, search, download, distribution, and lawful reuse in any medium only for non-commercial purposes, without prior permission from the Publisher or the author; provided the original work is properly cited and any changes to the original are clearly indicated. 